\title{
Plasma Xanthine Oxidoreductase Activity Associated with Glycemic Control in Patients with Pre-Dialysis Chronic Kidney Disease
}

\author{
Shinya Nakatani ${ }^{a}$ Eiji Ishimurab ${ }^{\text {Takayo Murase }}{ }^{c}$ Takashi Nakamurac $^{c}$ \\ Ayumi Nakatani $^{a}$ Norikazu Toi ${ }^{a}$ Kozo Nishide ${ }^{a}$ Hideki Uedono $^{a}$ \\ Akihiro Tsuda $^{a}$ Masafumi Kurajoha $^{a}$ Shinsuke Yamada ${ }^{a}$ Katsuhito Morib

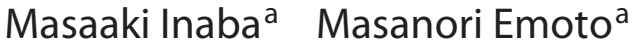 \\ aDepartment of Metabolism, Endocrinology and Molecular Medicine, Osaka City University Graduate School of \\ Medicine, Osaka, Japan; bepartment of Nephrology, Osaka City University Graduate School of Medicine, Osaka, \\ Japan; 'Mie Research Laboratories, Sanwa Kagaku Kenkyusho Co., Ltd., Inabe-shi, Japan
}

\section{Keywords}

Xanthine oxidoreductase activity · Chronic kidney disease · Diabetes mellitus

\begin{abstract}
Introduction: Xanthine oxidoreductase (XOR) activity plays an important role as a pivotal source of reactive oxygen species, which is associated with cardiovascular disease (CVD) events. Patients with CKD have increased risk of CVD events. In the present study, factors associated with plasma XOR activity in pre-dialysis CKD patients were investigated. Methods: In this cross-sectional study, plasma XOR activity in 118 pre-dialysis CKD patients (age 68 [57-75] years; 64 males, 26 with diabetes mellitus [DM]) was determined using a newly established highly sensitive assay based on $\left({ }^{13} \mathrm{C}_{2},{ }^{15} \mathrm{~N}_{2}\right)$ xanthine and liquid chromatography/triple quadrupole mass spectrometry. Results: Plasma glucose, hemoglobin A1C, and estimated glomerular filtration (eGFR) were significantly and positively correlated with plasma logarithmically transformed XOR (In-XOR) activity. In multiple regression analyses, eGFR and hemoglobin A1c or plasma glucose were significantly, independently, and positively associated with
\end{abstract}

karger@karger.com www.karger.com/kbr

Karger!

BOPEN ACCESS
(C) 2021 The Author(s)

Published by S. Karger AG, Basel

This is an Open Access article licensed under the Creative Commons Attribution-NonCommercial-4.0 International License (CC BY-NC) (http://www.karger.com/Services/OpenAccessLicense), applicable to the online version of the article only. Usage and distribution for commercial purposes requires written permission. plasma In-XOR activity after adjusting for several confounders. Plasma XOR activity was significantly higher in CKD patients with $(n=26)$ than in those without $(n=92) \mathrm{DM}(62.7$ [32.3-122] vs. $25.7[13.4-45.8] \mathrm{pmol} / \mathrm{h} / \mathrm{mL}, p<0.001)$. A total of 38 patients were taking uric acid-lowering drugs. Multiple regression analysis of CKD patients not administered uric acid-lowering drugs ( $n=80)$ showed no significant association between eGFR and plasma In-XOR activity. In contrast, association between glycemic control and plasma In-XOR activity was significant even in CKD patients without uric acidlowering drug treatment. Conclusions: These results indicate the importance of glycemic control in CKD patients in regard to decreased $X O R$, possibly leading to a decrease in CVD events.

(C) 2021 The Author(s)

Published by S. Karger AG, Basel

\section{Introduction}

$\mathrm{CKD}$ is strongly associated with cardiovascular disease (CVD), while a lower estimated glomerular filtration rate (eGFR) has been recognized as a strong and independent risk factor of CVD $[1,2]$. Although the high rates of mor- 
bidity and mortality related to CVD in patients with CKD are serious issues, they cannot be explained by traditional CVD risk factors alone, such as hypertension, diabetes mellitus (DM), smoking habit, or hypercholesterolemia [3] as oxidative stress is also involved in CVD development in those patients [3-5]. Xanthine oxidoreductase (XOR) is a rate-limiting enzyme that catalyzes formation of uric acid by oxidative hydroxylation of hypoxanthine and xanthine in purine metabolism [6], and its activation can increase reactive oxygen species, leading to oxidative stress-induced injury in various tissues [7-9]. Plasma $\mathrm{XOR}$ activity has been reported to be a predictor of CVD events in patients with pre-dialysis CKD as well as endstage kidney disease [10]. Thus, determination of plasma XOR activity is clinically important for patients with CKD.

To date, XOR activity assays have been based on determining formation of uric acid from xanthine as a substrate using an ultraviolet detector $[11,12]$. However, that traditional measurement method is not considered suitable for measuring uric acid-rich specimens such as human plasma from CKD patients because subtraction of the basal level of uric acid originally contained in the sample is inevitable [13]. In addition, XOR activity is extremely low in humans as compared with that in rodents, making accurate measurement difficult [14].

We recently developed a novel, accurate, and highly sensitive assay to measure XOR activity in human plasma, which utilizes stable isotope-labeled $\left({ }^{13} \mathrm{C}_{2},{ }^{15} \mathrm{~N}_{2}\right)$ xanthine as a substrate and liquid chromatography/mass spectrometry (LC/MS), composed of a Nano Space SI-2 LC system and TSQ-quantum triple quadrupole mass spectrometer (TQMS) [15]. Findings obtained with this novel assay have shown that plasma XOR activity is associated with severity and clinical outcome in patients with chronic heart failure [16], as well as left ventricular ejection fraction in patients with heart disease [17]. In our previous study, plasma glucose was demonstrated to be significantly associated with plasma XOR activity in hemodialysis patients [18]. However, knowledge regarding plasma XOR activity as a biomarker in pre-dialysis CKD patients is limited $[19,20]$, while the association of plasma XOR activity with various CKD-associated parameters has not been investigated. We report here results of a cross-sectional single-center investigation of 118 pre-dialysis CKD patients, in whom plasma XOR activity was measured using our newly developed method, as well as findings showing a potential relationship among CKDassociated parameters, including hemoglobin Alc, and plasma XOR activity.

\section{Materials and Methods}

\section{Patients}

CKD was defined according to criteria proposed by the Kidney Disease: Improving Global Outcomes Organization [21]. Patients diagnosed with CKD were regularly followed up by nephrologists at the departments of nephrology, metabolism, endocrinology, and molecular medicine at Osaka City University Hospital, of whom 118 examined from March to June 2016 were enrolled. The etiological factors included hypertensive nephrosclerosis $(n=29)$, diabetic nephropathy $(n=26)$, IgA nephropathy $(n=15)$, membranous nephropathy $(n=15)$, anti-neutrophil cytoplasmic antibody-associated glomerulonephritis $(n=5)$, autosomal dominant polycystic kidney disease $(n=4)$, minimal change nephrotic syndrome $(n=3)$, tubulointerstitial nephritis $(n=3)$, membranoproliferative glomerulonephritis $(n=2)$, focal and segmental glomerulosclerosis $(n=2)$, lupus nephritis $(n=1)$, and IgA vasculitis $(n=1)$, as well as unknown causes $(n=12)$. Those with hepatitis $\mathrm{B}$, hepatitis $\mathrm{C}$, malignancy, or a clinically overt infection were excluded. Thirty-eight of the 118 CKD patients enrolled were undergoing treatment with uric acid-lowering drugs or an XOR inhibitor, that is, allopurinol or febuxostat.

\section{Blood Sampling}

Spot blood and urine samples were collected from all patients in the morning after overnight fasting. The urine samples were kept on ice for $1 \mathrm{~h}$ and then centrifuged at $630 \mathrm{~g}$ for $10 \mathrm{~min}$, as previously described [22]. All laboratory measurements were performed using routine assays with automated methods [23]. eGFR was calculated using the new Japanese coefficient for the abbreviated modification of diet in renal disease study equation, with a correction factor of 0.739 used for females [24]. Serum calcium was corrected based on serum albumin and determined as corrected calcium, as previously described [23]. Serum intact parathyroid hormone (PTH) was measured using a second-generation Elecsys PTH IRMA assay (Roche Diagnostics, Mannheim, Germany), as previously reported [23]. Fibroblastic growth factor 23 (FGF-23), a bone-derived hormone, has a physiological role in regulation of phosphate, 1,25-dihydroxyvitamin D, and PTH [25-28]. Elevated FGF-23 levels have been shown to be associated with CVD events in patients with CKD $[29,30]$. It is conceivable that FGF-23 is a potential biomarker that may be useful to improve current clinical practice related to management of CKD mineral and bone disorder (CKD-MBD) [31-33]. Serum full-length FGF-23 was determined using a CL-JACK System (Kyowa Medex Co. Ltd., Tokyo, Japan), a fully automated random access chemiluminescence immunoanalyzer, as previously reported [22]. Plasma samples were kept in a freezer at $-80^{\circ} \mathrm{C}$ until determination of XOR activity.

\section{Determination of Plasma XOR Activity}

Plasma XOR activity was determined using freshly thawed samples that had been stored at $-80^{\circ} \mathrm{C}$ until the time of the assay with our recently established assay using stable isotope-labeled $\left({ }^{13} \mathrm{C}_{2},{ }^{15} \mathrm{~N}_{2}\right)$ xanthine with LC/MS (Nano Space SI-2 LC system, Shiseido, Tokyo, Japan, and TQMS, Thermo Fisher Scientific $\mathrm{GmbH}$, Bremen, Germany), as previously described $[14,15]$. In brief, $100-\mu \mathrm{L}$ aliquots of plasma were purified on a Sephadex G25 column and then mixed with Tris buffer ( $\mathrm{pH} 8.5$ ) containing $\left({ }^{13} \mathrm{C}_{2},{ }^{15} \mathrm{~N}_{2}\right)$ xanthine as the substrate and $\mathrm{NAD}^{+}$, with $\left({ }^{13} \mathrm{C}_{3},{ }^{15} \mathrm{~N}_{3}\right)$ uric acid used as an internal standard. The mixtures 
were incubated at $37^{\circ} \mathrm{C}$ for $90 \mathrm{~min}$, then methanol $(500 \mu \mathrm{L})$ was added and centrifugation was performed at 2,000 $\mathrm{g}$ for $15 \mathrm{~min}$ at $4^{\circ} \mathrm{C}$. Next, the supernatants were transferred to new tubes and dried using a centrifugal evaporator. The residues were reconstituted in $150 \mu \mathrm{L}$ of distilled water, filtered through an ultrafiltration membrane, and measured using LC/TQMS. Calibration standard samples were measured for $\left({ }^{13} \mathrm{C}_{2},{ }^{15} \mathrm{~N}_{2}\right)$ uric acid, and the amounts of $\left({ }^{13} \mathrm{C}_{2},{ }^{15} \mathrm{~N}_{2}\right)$ uric acid produced were calculated based on the calibration curve. XOR activity is expressed as $\mathrm{pmol} / \mathrm{mL} / \mathrm{h}$ of $\left({ }^{13} \mathrm{C}_{2},{ }^{15} \mathrm{~N}_{2}\right)$ uric acid produced. The intra- and inter-assay coefficients of variation were 6.5 and $9.1 \%$, respectively [15].

\section{Statistical Analysis}

Statistical analyses were performed using GraphPad Prism for Windows, version 6.0 (GraphPad Software, San Diego, CA), and the JMP software package, version 10 (SAS Institute Inc., Cary, $\mathrm{NC}$ ). Values are expressed as mean $\pm \mathrm{SD}$. Comparisons between CKD patients with and without uric acid-lowering drug administration, and with and without DM were made using unpaired Student's $t$-test or Mann-Whitney $U$ test for continuous variables, while a $\chi^{2}$ test was used for categorical variables. Plasma XOR activity was natural logarithm transformed $(\ln )$ to normalize the skewed distribution. Correlations between plasma logarithmically transformed XOR (ln-XOR) activity and clinical data were examined by Pearson's analysis. Independent associations between the variables and XOR activity in CKD patients were assessed by multiple regression analysis. Multiple regression analysis conducted previously showed that glycated albumin, a marker of glycemic control, was independently associated with plasma XOR activity in diabetic hemodialysis patients [18]. When plasma glucose was added instead of glycated albumin, those analysis findings showed that it was also independently associated with plasma XOR activity. Thus, hemoglobin Alc (model 1) and plasma glucose (model 2 ) were separately included as explanatory variables in the present multiple regression analyses. $p$ values $<0.05$ were considered to indicate statistical significance. Datasets generated and/or analyzed during the current study are available from the corresponding author upon reasonable request.

\section{Results}

\section{Clinical Characteristics of CKD Patients}

The clinical characteristics of the 118 CKD patients enrolled in this study are shown in Table 1. The median value [interquartile range] for eGFR, and serum creatinine levels were 31.7 [16.1-45.1] $\mathrm{mL} / \mathrm{min} / 1.73 \mathrm{~m}^{2}$, and $1.59[1.19-2.79] \mathrm{mg} / \mathrm{dL}$, respectively, while the mean $( \pm \mathrm{SD})$ value for uric acid in serum was $6.7 \pm 1.5 \mathrm{mg} / \mathrm{dL}$. Plasma XOR activity in all CKD patients was 29.2 [14.2$65.1] \mathrm{pmol} / \mathrm{h} / \mathrm{mL}$, lower than the mean $( \pm \mathrm{SD})$ value previously reported for healthy subjects $(89.1 \pm 55.1 \mathrm{pmol} / \mathrm{h} /$ $\mathrm{mL}, n=20)$ [15], but higher than that shown in patients undergoing hemodialysis $(21.4 \pm 13.5 \mathrm{pmol} / \mathrm{h} / \mathrm{mL}, n=$ 163) [18].

Xanthine Oxidoreductase Activity and Glycemic Control in CKD

Table 1. Clinical characteristics of CKD patients $(n=118)$

\section{Clinical variable}

Male/female

DM/non-DM

Age, years

$\mathrm{BMI}, \mathrm{kg} / \mathrm{m}^{2}$

eGFR, $\mathrm{mL} / \mathrm{min} / 1.73 \mathrm{~m}^{2}$

Creatinine, $\mathrm{mg} / \mathrm{dL}$

BUN, mg/dL

Total protein, $\mathrm{g} / \mathrm{dL}$

Albumin, g/dL

Alanine transaminase, IU/L

Aspartate transaminase, IU/L

Uric acid, $\mathrm{mg} / \mathrm{dL}$

Hemoglobin, g/dL

Iron, $\mu \mathrm{g} / \mathrm{dL}$

Ferritin, ng/mL

Plasma glucose, $\mathrm{mg} / \mathrm{dL}$

Hemoglobin A1c, \%

CRP, mg/L

Phosphate, $\mathrm{mg} / \mathrm{dL}$

Corrected calcium, mg/dL

Intact PTH, pg/mL

FGF-23, pg/mL

Urinary protein, $\mathrm{g} / \mathrm{gCr}$

$\mathrm{XOR}, \mathrm{pmol} / \mathrm{h} / \mathrm{mL}$

Uric acid-lowering drug administration

(allopurinol/febuxostat)

\author{
$64 / 54$ \\ $26 / 92$ \\ $68[57-75]$ \\ $21.6[19.5-25.0]$ \\ $31.7[16.1-45.1]$ \\ $1.59[1.19-2.79]$ \\ $24.5[19.0-38.3]$ \\ $6.7 \pm 0.66$ \\ $3.8[3.5-4.0]$ \\ $14[10-20]$ \\ $20[16-23]$ \\ $6.7 \pm 1.5$ \\ $12.6 \pm 1.77$ \\ $88.4 \pm 29.4$ \\ $83.6[34.5-169]$ \\ $99[87-117]$ \\ $5.6[5.4-6.1]$ \\ $0.06[0.02-0.16]$ \\ $3.6[3.3-4.3]$ \\ $9.5[9.2-9.7]$ \\ 70.5 [38.6-1 26] \\ $73[51-113]$ \\ $1.12[0.25-3.22]$ \\ $29.2[14.2-65.1]$
}

$38(13 / 25)$

Data are expressed as mean \pm SD. Median values [interquartile range] are shown for variables with skewed distributions. CRP, C-reactive protein; DM, diabetes mellitus; eGFR, estimated glomerular filtration rate; FGF-23, fibroblast growth factor-23; PTH, parathyroid hormone; XOR, xanthine oxidoreductase.

Correlations of Plasma ln-XOR Activity with Clinical Parameters in Pre-Dialysis CKD Patients

Correlations between various clinical parameters and plasma $\ln$-XOR activity were determined using simple regression analyses. In the present CKD patients, alanine transaminase, aspartate transaminase, eGFR (Fig. 1A), hemoglobin A1c (Fig. 1B), and plasma glucose (Fig. 1C) were each significantly and positively correlated with plasma $\ln$-XOR activity $(r=0.204, p=0.027$; $r=0.217, p=0.018 ; r=0.274, p=0.003 ; r=0.269, p=$ 0.003 ; and $r=0.384, p<0.001$, respectively), while serum creatinine, BUN, phosphate, and FGF-23 were significantly and negatively correlated with that activity $(r=$ $-0.270, p=0.003 ; r=-0.342, p<0.001 ; r=-0.224, p=$ 0.019 ; and $r=-0.222, p=0.017$, respectively) (Table 2). eGFR was significantly and positively correlated with plasma ln-XOR activity in the pre-dialysis CKD patients 


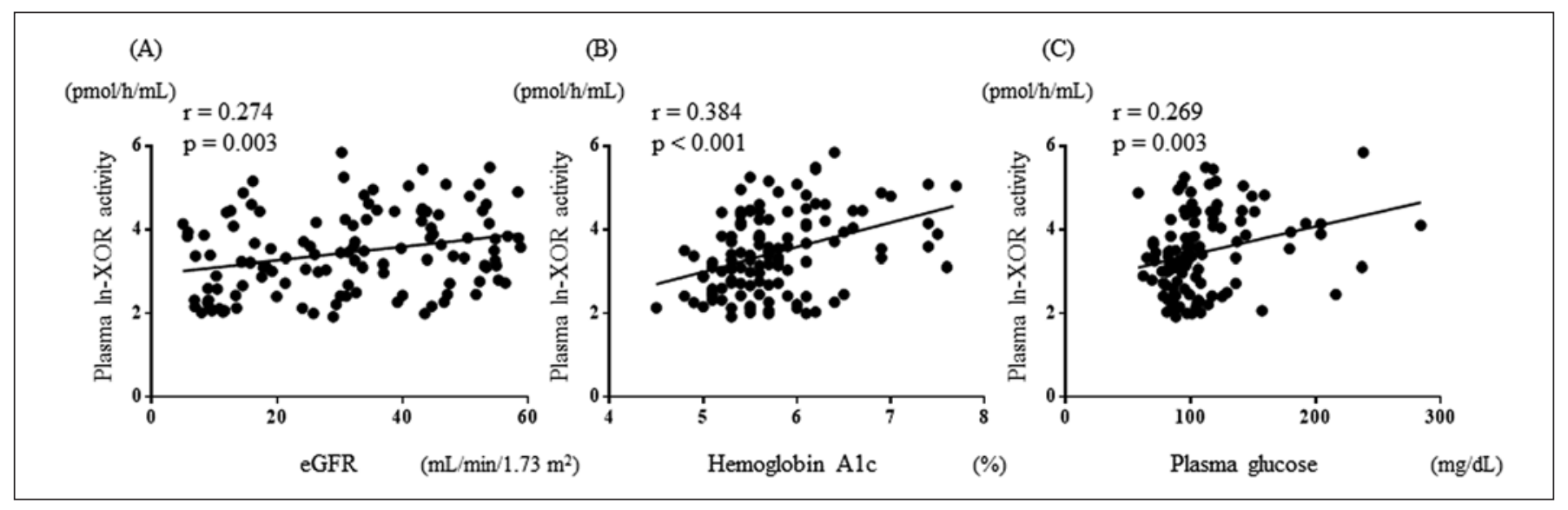

Fig. 1. Correlations between plasma $\ln -\mathrm{XOR}$ activity and clinical parameters in pre-dialysis CKD patients. eGFR (A), hemoglobin A1c (B), and plasma glucose were significantly (C) positively correlated with plasma ln-XOR activity. eGFR, estimated glomerular filtration rate; XOR, xanthine oxidoreductase; ln-XOR, logarithmically transformed XOR.

receiving uric acid-lowering drugs $(n=38, r=0.376 p=$ $0.020)$ as well as in those not receiving those drugs $(n=$ $80, r=0.224 p=0.046$ ) (online suppl. Fig. 1; see www. karger.com/doi/10.1159/000516610 for all online suppl. material).

\section{Association of Various CKD Parameters with Plasma In-XOR Activity}

Next, the associations of various CKD clinical parameters with plasma $\ln$-XOR activity were examined. In multiple regression analysis results, the variables age, gender, eGFR, uric acid, aspartate transaminase, FGF23 , and presence of diabetes were included as explanatory variables. The results showed that presence of diabetes was significantly and positively associated with plasma ln-XOR activity, independent of the other confounders. Thus, for multiple regression analysis, hemoglobin A1c was included as an explanatory variate in model 1 and plasma glucose was included in model 2 (Table 3).

In the present CKD patients, eGFR $(\beta=0.223, p=$ $0.028)$ and hemoglobin $A 1 c(\beta=0.332, p<0.001)$ were significantly and independently associated with plasma ln-XOR activity $\left(R^{2}=0.261, p<0.001\right)$ (Table 3, model $1)$, as were $\operatorname{eGFR}(\beta=0.281, p=0.007)$ and plasma glucose $(\beta=0.250, p=0.007)\left(R^{2}=0.222, p<0.001\right)$ (Table 3, model 2). In contrast, age, gender, BMI, serum uric acid, aspartate transaminase, and the FGF-23 level did not have a significant association with plasma $\ln$-XOR activity in the present CKD patients.
Table 2. Correlations between clinical parameters and plasma lnXOR activity in all patients (simple regression analyses; $n=118$ )

\begin{tabular}{|c|c|c|}
\hline \multirow[t]{2}{*}{ Clinical variable } & \multicolumn{2}{|c|}{$\begin{array}{l}\text { Correlation with plasma } \\
\text { ln-XOR activity }\end{array}$} \\
\hline & $r$ & $p$ value \\
\hline Age, years & 0.166 & 0.072 \\
\hline BMI, $\mathrm{kg} / \mathrm{m}^{2}$ & 0.111 & 0.229 \\
\hline $\mathrm{eGFR}, \mathrm{mL} / \mathrm{min} / 1.73 \mathrm{~m}^{2}$ & 0.274 & $0.003 *$ \\
\hline Creatinine, mg/dL & -0.270 & $0.003^{*}$ \\
\hline $\mathrm{BUN}, \mathrm{mg} / \mathrm{dL}$ & -0.342 & $<0.001^{*}$ \\
\hline Total protein, $\mathrm{g} / \mathrm{dL}$ & -0.096 & 0.303 \\
\hline Albumin, $\mathrm{g} / \mathrm{dL}$ & -0.092 & 0.321 \\
\hline Alanine transaminase, IU/L & 0.204 & $0.027^{*}$ \\
\hline Aspartate transaminase, IU/L & 0.217 & $0.018^{*}$ \\
\hline Uric acid, mg/dL & 0.032 & 0.733 \\
\hline Hemoglobin, g/dL & 0.066 & 0.477 \\
\hline Iron, $\mu \mathrm{g} / \mathrm{dL}$ & -0.001 & 0.942 \\
\hline Ferritin, ng/mL & -0.092 & 0.330 \\
\hline Plasma glucose, $\mathrm{mg} / \mathrm{dL}$ & 0.269 & $0.003^{*}$ \\
\hline Hemoglobin A1c, \% & 0.384 & $<0.001^{*}$ \\
\hline $\mathrm{CRP}, \mathrm{mg} / \mathrm{L}$ & 0.098 & 0.303 \\
\hline Phosphate, $\mathrm{mg} / \mathrm{dL}$ & -0.224 & $0.019 *$ \\
\hline Corrected calcium, mg/dL & 0.103 & 0.281 \\
\hline Intact PTH, pg/mL & -0.068 & 0.481 \\
\hline FGF-23, pg/mL & -0.222 & $0.017^{*}$ \\
\hline Urinary protein, $\mathrm{g} / \mathrm{gCr}$ & -0.059 & 0.526 \\
\hline
\end{tabular}

BMI, body mass index; BUN, blood urea nitrogen; CRP, Creactive protein; eGFR, estimated glomerular filtration rate; FGF23, fibroblast growth factor-23; PTH, parathyroid hormone; XOR, xanthine oxidoreductase; ln-XOR, logarithmically transformed XOR. Data include simple correlation coefficient $(r)$ and the level of significance $(p) .{ }^{*} p<0.05$. 
Clinical Parameters Associated with Plasma ln-XOR Activity in Pre-Dialysis CKD Patients without DM

Plasma XOR activity levels were significantly higher in CKD patients with $(n=26)$ than in those without $(n=92)$ $\mathrm{DM}(62.7$ [32.3-122] vs. 25.7 [13.4-45.8] $\mathrm{pmol} / \mathrm{h} / \mathrm{mL}, p<$ 0.001) (Fig. 2A). While the presence of diabetes can be related to increased plasma XOR activity, the number of CKD patients with DM was relatively small in the present study. Therefore, we performed multiple regression analyses to elucidate parameters associated with plasma lnXOR activity in those without DM, which showed that eGFR $(\beta=0.255, p=0.028)$ and hemoglobin Alc $(\beta=$ $0.251, p=0.023$ ) were significantly and independently associated with plasma ln-XOR activity in CKD patients without DM $\left(R^{2}=0.285, p<0.001\right)$ (Table 4 , model 1$)$. Furthermore, eGFR $(\beta=0.337, p=0.004)$ and plasma glucose $(\beta=0.245, p=0.019)$ were significantly and independently associated with plasma $\ln$-XOR activity in predialysis CKD patients without $\mathrm{DM}\left(R^{2}=0.288, p<0.001\right)$, while uric acid was also significantly and independently associated with plasma ln-XOR activity in those patients $(\beta=0.227, p=0.032)$ (Table 4 , model 2$)$.

\section{Plasma ln-XOR Activity in Pre-Dialysis CKD \\ Patients with and without Uric Acid-Lowering Drug Administration}

Uric acid-lowering drugs such as allopurinol or febuxostat are known to be XOR inhibitors and are being used by 38 of the present CKD patients. eGFR was not significantly different between patients with and those without such administration $(n=80)(30.1$ [8.89-44.7] vs. $\left.32.4[16.2-46.1] \mathrm{mL} / \mathrm{min} / 1.73 \mathrm{~m}^{2}, p=0.338\right)$, whereas the plasma XOR activity level was significantly lower in patients with than that in patients without such administration (11.7 [8.48-28.0] vs. 40.6 [22.6-83.9] $\mathrm{pmol} / \mathrm{h} / \mathrm{mL}, p<0.001$ ) (Fig. 2B). Since uric acid-lowering drugs are known to inhibit XOR activity, we performed multiple regression analysis to further identify parameters independently associated with plasma lnXOR activity in pre-dialysis CKD patients who were not taking uric acid-lowering drugs (Table 5). Age $(\beta=$ $0.221, p=0.014)$, aspartate transaminase $(\beta=0.327$, $p<0.001)$, and hemoglobin A1c $(\beta=0.368, p<0.001)$ were significantly and independently associated with plasma $\ln$-XOR activity $\left(R^{2}=0.475, p<0.001\right)$, while eGFR did not have an association in that model (Table 5 , model 1 ). When plasma glucose was included instead of hemoglobin A1c (model 2$)$, age $(\beta=0.192, p=$ $0.045)$, male gender $(\beta=-0.268, p=0.007)$, aspartate transaminase $(\beta=0.318, p=0.001)$, and plasma glucose

Xanthine Oxidoreductase Activity and Glycemic Control in CKD
Table 3. Results of multivariate analysis to elucidate various CKD parameters associated with plasma $\ln$-XOR activity in all patients $(n=118)$

\begin{tabular}{|c|c|c|c|c|}
\hline & \multicolumn{2}{|c|}{ Model 1} & \multicolumn{2}{|c|}{ Model 2} \\
\hline & $\beta$ & $p$ value & $\beta$ & $p$ value \\
\hline Age, years & 0.103 & 0.246 & 0.147 & 0.101 \\
\hline Gender & 0.106 & 0.237 & 0.102 & 0.267 \\
\hline BMI & 0.098 & 0.268 & 0.097 & 0.285 \\
\hline eGFR & 0.223 & 0.028 & 0.281 & 0.007 \\
\hline Uric acid & 0.148 & 0.102 & 0.140 & 0.131 \\
\hline Aspartate transaminase & 0.100 & 0.274 & 0.067 & 0.484 \\
\hline FGF-23 & -0.080 & 0.384 & -0.136 & 0.151 \\
\hline Hemoglobin A1c & 0.332 & $<0.001$ & - & - \\
\hline Plasma glucose & - & - & 0.250 & 0.007 \\
\hline$R^{2}$ & \multicolumn{2}{|c|}{$\begin{array}{l}0.261 \\
(p<0.001)\end{array}$} & \multicolumn{2}{|c|}{$\begin{array}{l}0.222 \\
(p<0.001)\end{array}$} \\
\hline
\end{tabular}

BMI, body mass index; eGFR, estimated glomerular filtration rate; FGF-23, fibroblast growth factor-23; XOR, xanthine oxidoreductase; $\beta$, standardized correlation coefficient; $R^{2}$, multiple coefficients of determination; ln-XOR, logarithmically transformed XOR.

$(\beta=0.307 p=0.002)$ were significantly and independently associated with plasma ln-XOR activity $\left(R^{2}=\right.$ $0.436, p<0.001)$.

\section{Discussion/Conclusion}

Clinical parameters associated with plasma XOR activity in pre-dialysis CKD patients were investigated, with measurements of plasma XOR activity performed using a newly established highly sensitive assay based on $\left({ }^{13} \mathrm{C}_{2},{ }^{15} \mathrm{~N}_{2}\right)$ xanthine and LC/TQMS. The presence of diabetes, hemoglobin A1c, plasma glucose, and eGFR were found to be significantly and independently associated with plasma ln-XOR activity after adjusting for other confounders. Notably, these associations between glycemic control and plasma ln-XOR activity were significant even in CKD patients without DM. As for CKD patients not taking uric acid-lowering drugs, the association between eGFR and plasma XOR activity was not significant, whereas the association between glycemic control and plasma XOR activity was significant in that subgroup.

In previous studies of DM and XOR activity, hepatic activity was 1.6-fold greater and the plasma XOR level was 3.7-fold higher in streptozotocin-induced diabetic rodents as compared with the respective control groups $[34,35]$. 


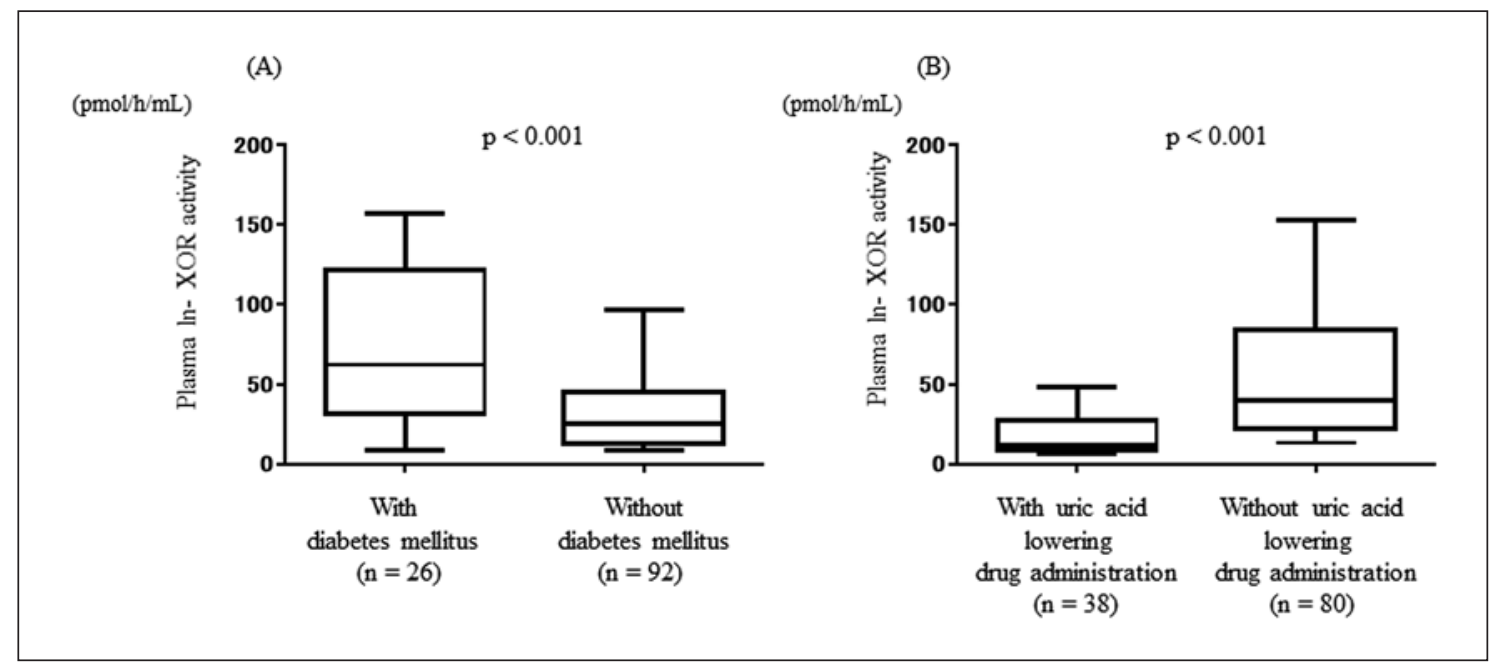

Fig. 2. Plasma XOR activity shown by box plots in patients with and without DM (A), and patients with and without administration of uric acid-lowering drugs (B). Box plots indicate 25th, 50th, and 75th percentile levels. A Plasma XOR activity was significantly higher in pre-dialysis CKD patients with than in those without DM. B Plasma XOR activity was significantly higher in pre-dialysis CKD patients without than in those with administration of uric acid-lowering drugs. XOR, xanthine oxidoreductase; DM, diabetes mellitus. ${ }^{*} p<0.05$.

Table 4. Results of multivariate analysis to elucidate various CKD parameters associated with plasma ln-XOR activity in pre-dialysis CKD patients without DM $(n=92)$

\begin{tabular}{|c|c|c|c|c|}
\hline & \multicolumn{2}{|c|}{ Model 1} & \multicolumn{2}{|c|}{ Model 2} \\
\hline & $\beta$ & $p$ value & $\beta$ & $p$ value \\
\hline Age, years & 0.072 & 0.492 & 0.101 & 0.311 \\
\hline Gender & 0.150 & 0.162 & 0.191 & 0.072 \\
\hline BMI & 0.128 & 0.233 & 0.110 & 0.313 \\
\hline eGFR & 0.255 & 0.028 & 0.337 & 0.004 \\
\hline Uric acid & 0.181 & 0.096 & 0.227 & 0.032 \\
\hline Aspartate transaminase & 0.132 & 0.227 & 0.104 & 0.347 \\
\hline FGF-23 & -0.073 & 0.485 & -0.116 & 0.267 \\
\hline Hemoglobin A1c & 0.251 & 0.023 & - & - \\
\hline Plasma glucose & - & - & 0.245 & 0.019 \\
\hline$R^{2}$ & \multicolumn{2}{|c|}{$\begin{array}{l}0.285 \\
(p<0.001)\end{array}$} & \multicolumn{2}{|c|}{$\begin{array}{l}0.288 \\
(p<0.001)\end{array}$} \\
\hline
\end{tabular}

eGFR, estimated glomerular filtration rate; FGF-23, fibroblast growth factor-23; XOR, xanthine oxidoreductase; DM, diabetes mellitus; ln-XOR, logarithmically transformed XOR; $\beta$, standardized correlation coefficient; $R^{2}$, multiple coefficients of determination.

Furthermore, plasma XOR activity was recently found to be significantly and positively associated with hemoglobin Alc in patients with type $1 \mathrm{DM}(n=71, r=0.292, p=0.013)$ [36], while BMI, fasting plasma glucose, insulin, and hemoglobin Alc were shown to be significantly and posi-
Table 5. Results of multivariate analysis to elucidate various CKD parameters associated with plasma ln-XOR activity in pre-dialysis CKD patients not being administered for uric acid-lowering drugs $(n=80)$

\begin{tabular}{|c|c|c|c|c|}
\hline & \multicolumn{2}{|c|}{ Model 1} & \multicolumn{2}{|c|}{ Model 2} \\
\hline & $\beta$ & $p$ value & $\beta$ & $p$ value \\
\hline Age, years & 0.221 & 0.014 & 0.192 & 0.045 \\
\hline Gender & -0.185 & 0.056 & -0.268 & 0.007 \\
\hline BMI & 0.157 & 0.077 & 0.151 & 0.102 \\
\hline eGFR & 0.044 & 0.673 & 0.096 & 0.368 \\
\hline Uric acid & 0.065 & 0.507 & 0.008 & 0.937 \\
\hline Aspartate transaminase & 0.327 & $<0.001$ & 0.318 & 0.001 \\
\hline Hemoglobin A1c & 0.368 & $<0.001$ & - & - \\
\hline Plasma glucose & - & - & 0.307 & 0.002 \\
\hline$R^{2}$ & \multicolumn{2}{|c|}{$\begin{array}{l}0.475 \\
(p<0.001)\end{array}$} & \multicolumn{2}{|c|}{$\begin{array}{l}0.436 \\
(p<0.001)\end{array}$} \\
\hline
\end{tabular}

eGFR, estimated glomerular filtration rate; XOR, xanthine oxidoreductase; $\beta$, standardized correlation coefficient; $R^{2}$, multiple coefficients of determination; $\ln -\mathrm{XOR}$, logarithmically transformed XOR.

tively correlated with plasma XOR activity in healthy subjects not receiving any medication [37]. In the present study, plasma XOR activity was significantly greater in predialysis CKD patients with than in those without DM. Of importance, other clinical parameters including kidney 
function were not significantly different between the groups (online suppl. Table 1), suggesting that impaired glycemic control itself is an important factor related to increased plasma XOR activity. Patients with DM have insulin resistance $[38,39]$, which is known to impair glycolysis, resulting in activation of the pentose phosphate pathway, followed by promotion of a hepatic de novo purine synthetic pathway [40], thereby triggering increased XOR activity. Although the mechanism remains unknown, redundant fructose in diabetic patients may also increase XOR activity by increasing ATP degradation to AMP, leading to increased nucleotides, which are uric acid precursors [41].

Recently, plasma XOR activity was found to be associated with vascular endothelial dysfunction in patients with type $1 \mathrm{DM}$ [36]. In addition, elevated plasma XOR activity has been reported to be associated with increased CVD events in heart failure patients with preserved ejection function [42] and higher mortality in cardiovascular intensive care patients [43], as well as an independent predictor of CVD events in CKD and hemodialysis patients [10]. Interestingly, Kawachi et al. [44] demonstrated that short-term glycemic control treatment (2 weeks hospitalization) decreased plasma XOR activity in patients with type $2 \mathrm{DM}$. Together, findings showing an association between glycemic control and plasma XOR activity in patients with type 1 diabetes [36], patients under hemodialysis in our previous study [18], and pre-dialysis CKD patients in the present study suggest the importance of glycemic control in regard to decreased XOR, possibly leading to a decrease in CVD events.

The correlation between kidney function and plasma $X O R$ activity remains controversial. In a previous study in which plasma XOR activity was measured using a traditional method, Gondouin et al. [10] reported that increased XOR activity in accordance with a decrease in kidney function was seen in 51 pre-dialysis CKD patients as well as 50 other CKD patients undergoing hemodialysis. In contrast, a significant and inverse correlation between plasma $\ln$-XOR activity and kidney dysfunction was shown in the present study. Furthermore, when the correlation was examined separately in patients receiving or not receiving uric acid-lowering drugs, it remained significant in both groups. These significant correlations were consistent with findings presented in 3 other recent studies, in which plasma XOR activity was measured in our novel method. Otaki et al. [16] investigated plasma XOR activity in patients with chronic heart failure and found that in their lower eGFR group $(53 \pm 25 \mathrm{~mL} /$ $\mathrm{min} / 1.73 \mathrm{~m}^{2}$ ) to be significantly reduced as compared to the normal eGFR group $\left(68 \pm 26 \mathrm{~mL} / \mathrm{min} / 1.73 \mathrm{~m}^{2}\right)$. Ad-

Xanthine Oxidoreductase Activity and Glycemic Control in CKD ditionally, Shirakabe et al. [45] demonstrated that the serum creatinine level was independently associated with low plasma XOR activity in patients with acute heart failure, while Terawaki et al. [19] showed a strong correlation between plasma XOR activity and eGFR $(r=0.751, p=$ $0.002)$ in pre-dialysis CKD patients $(n=13)$. We speculate that this discrepancy may have been due to differences in measurement methods used to determine plasma XOR activity. With the traditional method, elevated endogenous uric acid in a CKD patient may influence elevated XOR activity, since the plasma XOR level is determined based on uric acid after addition of xanthine as a substrate to the sample. In contrast, our measurement method is not affected by endogenous uric acid in samples from CKD patients, since only isotope-labeled $\left({ }^{13} \mathrm{C}_{2},{ }^{15} \mathrm{~N}_{2}\right)$ xanthine is measured. Findings noted in previous studies [16, $19,45]$ obtained by use of our novel measurement method support the inverse correlation between plasma XOR activity and kidney dysfunction noted in the present study.

Although XOR plays a pivotal role in uric acid production, no significant association between the serum uric acid level and plasma ln-XOR activity was observed in the present study. Recent studies that used our method have found serum uric acid to be positively correlated with plasma XOR activity in healthy subjects [37, 46, 47]. In contrast, in pre-dialysis CKD patients examined using the same method, serum uric acid was shown to be negatively correlated with plasma $\ln$-XOR activity $(r=-0.562$, $p=0.043$ ) [19]. Since uric acid excretion is dependent on kidney function $[48,49]$, it is possible that its level in serum is affected by decreased urinary uric acid excretion in CKD patients, rather than XOR activity itself.

Regarding CKD-MBD, calcium, phosphate, and intact-PTH were not found to be correlated with plasma ln-XOR activity in patients with hemodialysis [18], though FGF-23, an important factor of CVD events in CKD patients [50], was not evaluated in that study. In the present results, FGF-23 was shown to be significantly and negatively correlated with plasmaln-XOR activity, whereas it did not have a significant association with plasma ln-XOR activity after adjusting for several confounders, suggesting that CKD-MBD status is not associated with plasma ln-XOR activity.

The present study has some limitations. First, the number of patients examined was relatively small mainly because all were enrolled from a single institution. Notably, there were few diabetic CKD patients in whom the association between glycemic control status and plasma XOR activity was to be thoroughly investigated as the present study participants were followed up by nephrologists, not 
by diabetologists. Nevertheless, hemoglobin A1c and plasma glucose were significantly and positively associated with plasma XOR activity after adjustments with several confounders in all CKD patients, even when a small number of diabetic patients were included. Additional largescale studies are needed to investigate whether glycemic control status is associated with plasma $\ln$-XOR activity in diabetic patients with or without CKD. Additionally, this study had a cross-sectional design, and the results did not demonstrate causality of the examined parameters, that is, poor glycemic control, associated with increased plasma $\mathrm{XOR}$ activity. Future studies are required to examine whether plasma XOR activity is reduced in pre-dialysis CKD patients by strict glycemic control, while any link between glycemic control and XOR activity should be confirmed using experimental animal models. In addition, in vitro studies may also be necessary to determine the potential effect of plasma glucose on XOR activity.

In conclusion, this is the first study to show a significant positive association of plasma XOR activity with hemoglobin A1c and plasma glucose levels in pre-dialysis CKD patients. The results suggest that glycemic control in CKD patients is important for decreased plasma XOR activity, leading to a decrease in CVD events.

\section{Acknowledgements}

The authors acknowledge the technical assistance of Dr. Masaya Sasagawa of the research laboratory at the Department of Metabolism, Endocrinology, and Molecular Medicine, Osaka City University Graduate School of Medicine.

\section{Statement of Ethics}

This study was approved by the Ethics Committee of Osaka City University Graduate School of Medicine (approval No. \#3366). All participants provided written informed consent for collection of both blood and urine samples, as well as the use of clinical records relevant to the study. The research was conducted in accordance with the Declaration of Helsinki.

\section{Conflict of Interest Statement}

S.N., A.N., and E.I. received a research grant from Sanwa Kagaku Kenkyusho Co., Ltd. (Inabe-shi, Mie, Japan). The other authors have no conflicts of interest to declare in regard to this study.

\section{Funding Sources}

The authors did not receive any funding.

\section{Author Contributions}

S.N. and E.I. contributed to study concept and design, and data acquisition, analysis, and interpretation. A.N. and N.T. contributed to data acquisition. T.M. and T.N. performed measurements. K.N., H.U., A.T., M.K., S.Y., K.M., M.I., and M.E. contributed to the study concept and interpretation. All authors have read and approved the submitted version of the manuscript.

\section{References}

1 Go AS, Chertow GM, Fan D, McCulloch CE, Hsu CY. Chronic kidney disease and the risks of death, cardiovascular events, and hospitalization. N Engl J Med. 2004;351: 1296-305.

2 Ninomiya T, Kiyohara Y, Kubo M, Tanizaki Y, Doi Y, Okubo K, et al. Chronic kidney disease and cardiovascular disease in a general Japanese population: the Hisayama Study. Kidney Int. 2005;68:228-36.

3 Wanner C, Amann K, Shoji T. The heart and vascular system in dialysis. Lancet. 2016;388: 276-84.

4 Shlipak MG, Fried LF, Cushman M, Manolio TA, Peterson D, Stehman-Breen C, et al. Cardiovascular mortality risk in chronic kidney disease: comparison of traditional and novel risk factors. JAMA. 2005;293:1737-45.

5 Himmelfarb J. Uremic toxicity, oxidative stress, and hemodialysis as renal replacement therapy. Semin Dial. 2009;22:636-43.
6 Battelli MG, Bolognesi A, Polito L. Pathophysiology of circulating xanthine oxidoreductase: new emerging roles for a multi-tasking enzyme. Biochim Biophys Acta. 2014;1842:1502-17.

7 Berry CE, Hare JM. Xanthine oxidoreductase and cardiovascular disease: molecular mechanisms and pathophysiological implications. J Physiol. 2004;555:589-606.

8 Battelli MG, Polito L, Bolognesi A. Xanthine oxidoreductase in atherosclerosis pathogenesis: not only oxidative stress. Atherosclerosis. 2014;237:562-7.

9 Kelley EE. A new paradigm for XOR-catalyzed reactive species generation in the endothelium. Pharmacol Rep. 2015;67:669-74.

10 Gondouin B, Jourde-Chiche N, Sallee M, Dou L, Cerini C, Loundou A, et al. Plasma xanthine oxidase activity is predictive of cardiovascular disease in patients with chronic kidney disease, independently of uric acid levels. Nephron. 2015;131:167-74.
11 Waud WR, Rajagopalan KV. Purification and properties of the NAD+-dependent (type $\mathrm{D}$ ) and $\mathrm{O} 2$-dependent (type $\mathrm{O}$ ) forms of rat liver xanthine dehydrogenase. Arch Biochem Biophys. 1976;172:354-64.

12 Cighetti G, Del Puppo M, Andreetta F, Kienle MG. Xanthine oxidase activity: simultaneous HPLC evaluation of the "D" and "O" forms. Biochem Int. 1989;18:1211-20.

13 Liu X, Lin WM, Yan XH, Chen XH, Hoidal JR, $\mathrm{Xu}$ P. Improved method for measurement of human plasma xanthine oxidoreductase activity. J Chromatogr B Analyt Technol Biomed Life Sci. 2003;785:101-14.

14 Murase T, Oka M, Nampei M, Miyachi A, Nakamura T. A highly sensitive assay for xanthine oxidoreductase activity using a combination of [(13) C2,(15) N2] xanthine and liquid chromatography/triple quadrupole mass spectrometry. J Labelled Comp Radiopharm. 2016;59:214-20. 
15 Murase T, Nampei M, Oka M, Miyachi A, Nakamura T. A highly sensitive assay of human plasma xanthine oxidoreductase activity using stable isotope-labeled xanthine and LC/ TQMS. J Chromatogr B Analyt Technol Biomed Life Sci. 2016;1039:51-8.

16 Otaki Y, Watanabe T, Kinoshita D, Yokoyama M, Takahashi T, Toshima T, et al. Association of plasma xanthine oxidoreductase activity with severity and clinical outcome in patients with chronic heart failure. Int J Cardiol. 2017;228:151-7.

17 Fujimura Y, Yamauchi Y, Murase T, Nakamura T, Fujita SI, Fujisaka T, et al. Relationship between plasma xanthine oxidoreductase activity and left ventricular ejection fraction and hypertrophy among cardiac patients. PLoS One. 2017;12:e0182699.

18 Nakatani A, Nakatani S, Ishimura E, Murase T, Nakamura T, Sakura M, et al. Xanthine oxidoreductase activity is associated with serum uric acid and glycemic control in hemodialysis patients. Sci Rep. 2017;7:15416.

19 Terawaki H, Murase T, Nakajima A, Aoyagi K, Fukushima N, Tani Y, et al. The relationship between xanthine oxidoreductase and xanthine oxidase activities in plasma and kidney dysfunction. J Clin Exp Nephrol. 2017; 2(1):31.

20 Terawaki H, Hayashi T, Murase T, Iijima R, Waki K, Tani Y, et al. Relationship between xanthine oxidoreductase redox and oxidative stress among chronic kidney disease patients. Oxid Med Cell Longev. 2018;2018:9714710.

21 Inker LA, Astor BC, Fox CH, Isakova T, Lash JP, Peralta CA, et al. KDOQI US commentary on the 2012 KDIGO clinical practice guideline for the evaluation and management of CKD. Am J Kidney Dis. 2014;63:713-35.

22 Nakatani S, Ishimura E, Mori K, Fukumoto S, Yamano S, Wei M, et al. Nephronectin expression in glomeruli of renal biopsy specimens from various kidney diseases: nephronectin is expressed in the mesangial matrix expansion of diabetic nephropathy. Nephron Clin Pract. 2012;122:114-21.

23 Kurajoh M, Inaba M, Yamada S, Imanishi Y, Tsuchida T, Ishimura E, et al. Association of increased active PTH(1-84) fraction with decreased GFR and serum $\mathrm{Ca}$ in predialysis $\mathrm{CRF}$ patients: modulation by serum $25-\mathrm{OH}-\mathrm{D}$. Osteoporos Int. 2008;19:709-16.

24 Matsuo S, Imai E, Horio M, Yasuda Y, Tomita K, Nitta K, et al. Revised equations for estimated GFR from serum creatinine in Japan. Am J Kidney Dis. 2009;53:982-92.

25 Imanishi Y, Inaba M, Nakatsuka K, Nagasue $\mathrm{K}$, Okuno S, Yoshihara A, et al. FGF-23 in patients with end-stage renal disease on hemodialysis. Kidney Int. 2004;65:1943-6.
26 Quarles LD. Endocrine functions of bone in mineral metabolism regulation. J Clin Invest. 2008; 118:3820-8

27 Silver J, Rodriguez M, Slatopolsky E. FGF23 and PTH--double agents at the heart of CKD. Nephrol Dial Transplant. 2012;27:1715-20.

28 Kuro OM. A phosphate-centric paradigm for pathophysiology and therapy of chronic kidney disease. Kidney Int Suppl (2011). 2013; 3(5):420-6.

29 Xue C, Yang B, Zhou C, Dai B, Liu Y, Mao Z, et al. Fibroblast growth factor 23 predicts allcause mortality in a dose-response fashion in pre-dialysis patients with chronic kidney disease. Am J Nephrol. 2017;45:149-59.

30 Block GA, Chertow GM, Cooper K, Xing S, Fouqueray B, Halperin M, et al. Fibroblast growth factor 23 as a risk factor for cardiovascular events and mortality in patients in the EVOLVE trial. Hemodial Int. 2021;25(1):7885.

31 Mazzaferro S, Tartaglione L, Rotondi S, Bover J, Goldsmith D, Pasquali M. News on biomarkers in CKD-MBD. Semin Nephrol. 2014; 34:598-611.

32 Tan SJ, Cai MM. Is there a role for newer biomarkers in chronic kidney disease-mineral and bone disorder management? Nephrology. 2017;22(Suppl 2):14-8.

33 Vervloet M. Renal and extrarenal effects of fibroblast growth factor 23. Nat Rev Nephrol. 2019;15:109-20.

34 Desco MC, Asensi M, Marquez R, MartinezValls J, Vento M, Pallardo FV, et al. Xanthine oxidase is involved in free radical production in type 1 diabetes: protection by allopurinol. Diabetes. 2002;51:1118-24.

35 Matsumoto S, Koshiishi I, Inoguchi T, Nawata H, Utsumi H. Confirmation of superoxide generation via xanthine oxidase in streptozotocin-induced diabetic mice. Free Radic Res. 2003;37:767-72.

36 Washio K, Kusunoki Y, Tsunoda T, Osugi K, Ohigashi M, Murase T, et al. Xanthine oxidoreductase activity correlates with vascular endothelial dysfunction in patients with type 1 diabetes. Acta Diabetol. 2020;57:31-9.

37 Furuhashi M, Matsumoto M, Murase T, Nakamura T, Higashiura Y, Koyama M, et al. Independent links between plasma xanthine oxidoreductase activity and levels of adipokines. J Diabetes Investig. 2019;10:1059-67.

38 Lillioja S, Mott DM, Spraul M, Ferraro R, Foley JE, Ravussin E, et al. Insulin resistance and insulin secretory dysfunction as precursors of non-insulin-dependent diabetes mellitus. Prospective studies of Pima Indians. N Engl J Med. 1993 30;329:1988-92.
39 Taylor R. Insulin resistance and type 2 diabetes. Diabetes. 2012;61:778-9.

40 Leyva F, Wingrove CS, Godsland IF, Stevenson JC. The glycolytic pathway to coronary heart disease: a hypothesis. Metab Clin Exp. 1998;47:657-62.

41 Choi HK, Willett W, Curhan G. Fructose-rich beverages and risk of gout in women. JAMA. 2010;304:2270-8.

42 Watanabe K, Watanabe T, Otaki Y, Shishido $\mathrm{T}$, Murase T, Nakamura T, et al. Impact of plasma xanthine oxidoreductase activity in patients with heart failure with preserved ejection fraction. ESC Heart Fail. 2020;7: 1735-43.

43 Shibata Y, Shirakabe A, Okazaki H, Matsushita M, Goda H, Shigihara S, et al. Plasma xanthine oxidoreductase (XOR) activity in patients who require cardiovascular intensive care. Heart Vessels. 2020;35:1390-400.

44 Kawachi Y, Fujishima Y, Nishizawa H, Nagao H, Nakamura T, Akari S, et al. Plasma xanthine oxidoreductase activity in Japanese patients with type 2 diabetes across hospitalized treatment. J Diabetes Investig. 2020. Epub ahead of print.

45 Shirakabe A, Okazaki H, Matsushita M, Shibata Y, Goda H, Shigihara S, et al. Characteristics of patients with an abnormally decreased plasma xanthine oxidoreductase activity in acute heart failure who visited the emergency department. Cardiology. 2020; 145:473-80.

46 Washio KW, Kusunoki Y, Murase T, Nakamura T, Osugi K, Ohigashi M, et al. Xanthine oxidoreductase activity is correlated with insulin resistance and subclinical inflammation in young humans. Metab Clin Exp. 2017;70: 51-6.

47 Furuhashi M, Matsumoto M, Tanaka M, Moniwa N, Murase T, Nakamura T, et al. Plasma xanthine oxidoreductase activity as a novel biomarker of metabolic disorders in a general population. Circ J. 2018;82:1892-9.

48 Kanbara A, Hakoda M, Seyama I. Urine alkalization facilitates uric acid excretion. Nutr J. 2010;9:45.

49 Filippatos GS, Ahmed MI, Gladden JD, Mujib M, Aban IB, Love TE, et al. Hyperuricaemia, chronic kidney disease, and outcomes in heart failure: potential mechanistic insights from epidemiological data. Eur Heart J. 2011;32: 712-20.

50 Kidney Disease: Improving Global Outcomes CKDMBDUWG. KDIGO 2017 clinical practice guideline update for the diagnosis, evaluation, prevention, and treatment of Chronic Kidney Disease-Mineral and Bone Disorder (CKD-MBD). Kidney Int Suppl. 2011;7:1-59. 